\title{
Hemp Fibre as an Alternative to Glass Fibre in Sheet Moulding Compound: Part 1. Influence of Fibre Content and Surface Treatment on Mechanical Properties
}

\author{
H.K. Patel ${ }^{\mathrm{a}}$, G. Ren ${ }^{\mathrm{b}}$, P.J. $\operatorname{Hogg}^{\mathrm{c}}$, T. Peijs $\mathrm{s}^{\mathrm{a}, \mathrm{d}}$ \\ ${ }^{a}$ School of Engineering and Materials Science, Centre for Materials Research, Queen Mary, University of \\ London, London E1 4NS, UK \\ ${ }^{\mathrm{b}}$ School of Aerospace, Automotive and Design Engineering, University of \\ Hertfordshire, Hatfield AL10 9AB, UK \\ ${ }^{\mathrm{c} N o r t h w e s t ~ C o m p o s i t e s}$ Centre, University of Manchester, Manchester M13 9PL, UK \\ ${ }^{d}$ Eindhoven Polymer Laboratories, Eindhoven University of Technology, P.O. Box513, 5600 MB \\ Eindhoven, The Netherlands \\ * Corresponding author. Tel.: +44-0207-8828865; fax: +44-0207-89819804 \\ E-mail address: t.peijs@qmul.ac.uk
}

\begin{abstract}
Hemp fibre mat reinforced polyester composites were fabricated using a conventional sheet moulding compound (SMC) process. The influence of fibre and $\mathrm{CaCO}_{3}$ filler content on strength and stiffness of the HF-SMCs is reported and compared with data for chopped glass fibre reinforced sheet moulding compounds (GF-SMCs). In addition the influence of alkaline and silane treatments of the hemp fibres is evaluated. The experimental data is compared to modified versions of the Cox-Krenchel and Kelly-Tyson models, supplemented with parameters of composite porosity to improve the prediction of composite tensile properties. A good agreement was found between the modified models and experimental data for strength and stiffness. The results indicate that HF-SMCs are of interest for lowcost engineering applications that require high stiffness to weight ratios.

Keywords: Hemp fibre, Glass fibre, Interface, Mechanical properties
\end{abstract}

\section{Introduction}

Sheet moulding compounds (SMCs) are composite materials that, due to there mechanical and aesthetical properties have been employed in a large numbers of applications from sport and automotive to building applications [1]. However, despite their numerous advantages there are some drawbacks associated with glass fibres used in SMC composites. They are non-renewable, non-biodegradable materials, and give 
problems with respect to ultimate disposal at the end of a materials lifetime. Even incineration cannot completely thermally decompose every component and the residues of glass fibre and fillers will be left behind which are damaging to incineration furnace [2]. Additionally these fibres are very abrasive which leads to increased tool wear therefore increasing overall life-cycle cost. Furthermore, glass fibres pose enormous health and safety issues environmentally, such as inhalation and skin irritations during handling of these products [3].

Some alternative fibre reinforcements for the SMC composites have been developed such as the use of hemp or flax natural fibres . Natural fibre composites (NFCs) can be recycled if a thermoplastic matrix is used or thermally incinerated with energy recovery in the case of a thermosetting matrix, since these fibres have a good calorific value compared to glass fibres [4]. Natural fibres have additional advantages which make them suitable as reinforcement in SMC composites such as low density and high specific mechanical properties [5-6]. Although natural fibres have many advantages, there are certain drawbacks which have restricted their use. Natural fibres have a high rate of moisture absorption $[3,7]$ that can affect the durability of the composite in outdoor applications as well as being susceptible to poor dimensional stability and rotting [8-9]. Moreover, micro-cracking of the composite resulting from fibre swelling can cause degradation of mechanical properties. NFCs tend to give low impact strength [10] and poor fire resistant properties [11]. Additionally, there is poor compatibility between the hydrophilic natural fibres and the often hydrophobic polymeric resins, which leading to weak interface, resulting in inferior mechanical performance [12] and increased water absorption [13] of the composite material. Finally, the drawbacks of natural fibres include seasonal, geological growth patterns and quality controls, which affected the supply chain and variability of the product quality.

The interface between fibre and matrix plays an important role in transferring the stresses acting on the matrix to the fibres. For a composite to have good mechanical properties the fibre-matrix interface must be relatively strong. However, if the interface is too strong the composite will fail in a brittle mode and have low toughness; therefore it is important to optimise the fibre-matrix interface to accomplish a balance between strength and toughness of the composite [14-17]. There have been many studies to improve the bonding between natural fibres and the polymer resin through different chemical methods $[18-24]$.

One of the most common methods of chemical modification of natural fibres is a treatment with an alkaline solution (also called mercerization), such as $\mathrm{NaOH}$, resulting in a change of fibre properties. 
Mercerization directly influences the cellulosic structure, increasing surface roughness of the fibres and resulting in better mechanical interlocking and increasing fibre wetting [18]. Improvements in mechanical performance have been reported by many researches after alkali treatment of natural fibres [18-21]. For example it was found that the flexural properties of polyester composites reinforced by sponge gourd fib retreated with 5\% alkali, gave the best flexural properties. Murkherjee et al. [23] found that the use of more than $1 \% \mathrm{NaOH}$ on cellulose fibres weakens the fibres resulting in a reduction in mechanical properties.

Silane coupling agents or sizing, another fibre surface modification method, have been used in glass fibres for reinforced polymeric composites for a long time and now they are also being evaluated to improve interface properties in wood and natural fibre composites (Ref). Many studies have reported the use of silane based coupling agents for natural fibres and found it to be effective in modifying the fibre/matrix interface. Herrera and Valadez [24] studied the effect of silane treatment on the mechanical behaviour of high density polyethylene reinforced with henequen fibres. It is found that silane coupling agents initially increased the tensile strength of the composite due to improved degree of fibre/matrix adhesion, while at high silane concentrations the tensile strength decreased. The flexural properties of epoxy composites reinforced by flax fibre with silane treatment was investigated [25] giving improved the tensile strength and modulus of the composites in both longitudinal and transverse directions after the silane treatment.

Recently, the interest in Thermoset as matrices for natural fibre composites has been increasing [26-30]. At present 37\% of all natural fibre reinforced composites produced in Europe are based on Thermoset matrices compared to $63 \%$ for thermoplastic. Thermoset matrices offer better mechanical properties and an improved fibre-matrix adhesion than thermoplastics. Additionally, it is important to understand that the use of a thermoset resin does not necessarily diminish the eco-performance of the produced composites considering they can be recycled through thermal incineration. In fact the use of thermosets could be more complimentary then thermoplastic resin as the addition of natural fibre will considerably lower the mechanical recyclability of the thermoplastic also thermosets lower the energy required at manufacturing as they are normally cured at lower temperatures. Bio-based composites are potentially more eco-friendly, however the current biodegradable polymers are often of modest strength and not cost-effective at present [31-32]. A relatively new approach is to use cellulose as the matrix material which is reinforced by cellulose or plant fibres together to create all-cellulose composites 
$[21,33]$. Natural fibres are nowadays also available in yarn form or as textile reinforcement. Even though textile natural fibre reinforced composites offer better mechanical performance then non-woven's [2,3034] their price is considerably higher also the embodied energy is considerably increased by the necessary spinning and weaving stages. Therefore non-woven hemp mats are used in the present investigation.

In this study an attempt has been made to use hemp fibre as a principal reinforcement in SMC composite. The composite with a range of fibre volume fractions and calcium carbonate $\left(\mathrm{CaCO}_{3}\right)$ filler content was made to determine the optimum fibre and filler content for mechanical performance. To further improve the mechanical properties of the composite material, hemp fibres were treated with either $\mathrm{NaOH}$ or silane. The composite materials are mechanically tested in tension to determine their strength and Young's modulus. The fracture surface of the tested samples was analysed with SEM technique to understand the failure mechanisms of the composites.

\section{Experimental works}

\subsection{Materials}

Randomly oriented non-woven hemp mat were purchased from Hemcore Limited with an areal density of $500 \mathrm{~g} / \mathrm{m}^{2}$ and an average fibre length of $65 \pm 15 \mathrm{~mm}$. An orthophthalic unsaturated polyester (UP) resin (DSM Resins, UK) with standard fillers and additives was used as resin system. Standard SMC E-glass roving (Tex2400, OCF) was used for the standard glass fibre SMC as a reference or control material. Calcium carbonate $\left(\mathrm{CaCO}_{3}\right)$ with a particle size of $10 \mu \mathrm{m}$ was used as major filler. Some of the SMC resin system or paste, E-glass roving, calcium carbonate filler and GF-SMC were provided by Menzolit Ltd, UK. Methyl ethyl ketone peroxide catalyst, p-Benzoquinone polymerisation inhibitor, zinc stearate thickener and butylated hydroxytoluene initiator were also provided by Menzolit Lt, UK. The silane used was acetoxysilane Z-6075 supplied by Dow Corning. Laboratory reagent grade $\mathrm{NaOH}$ pellets were purchased from Fisher Scientific.

\subsection{Fibre surface treatment}

Before treatment the hemp fibre mats were first dried in an oven at $50^{\circ} \mathrm{C}$ for 24 hours.

Alkaline treatment: Fibre mats were immersed in $\mathrm{NaOH}$ solution (0.5-5 wt.\%) for 1 hour and then washed with distilled water for several times. Finally, the fibre mats were washed with distilled water containing $0.5 \%$ of $\mathrm{HCl}$. The washed fibres were then dried at $80^{\circ} \mathrm{C}$ for 5 hours. Silane treatment: Fibre mat was immersed in a water/acetone mixture (5:95 wt.\%) containing a silane coupling agent (0.5-5 wt.\%). The 
fibre mat was then washed with distilled water and dried in an oven at $80^{\circ} \mathrm{C}$ for 5 hours. Alkaline-Silane treatment: Fibre mat was first treated with 2 wt.\% alkaline solution and then treated with silane $(0.5-5$ wt.\%).

\subsection{Composite fabrication}

Hemp fibre reinforced composites were prepared by a conventional SMC machine shown in Figure 1. The SMC machine comprises of two stations for unwinding polyamide film rolls which served as a support for the compounds. To make the compound, first an impregnation paste was fed into the bottom resin box of the machine. The SMC manufacturing started by moving the bottom and top films along the machine as the resin paste was deposited on both films with uniform thickness by means of two doctor blades with an adjustable height. Simultaneously, the hemp fibre mat roll was fed in between the two films, producing a sandwich film structure then moved along the machine between a series of compaction rollers. This SMC material sandwiched by two films was pressed through number of rollers for forming a fully mixed layer of resin paste against the hemp fibre mat. The hemp prepreg was then left in a store room at elevated temperature around 30-40C for a number of days for viscosity incensement called SMC thickening process (up to $1.0 \times 10^{6} \mathrm{cps}$ ). The thickened material was then press moulded in a hot press using a mould of $300 \mathrm{~mm} \times 300 \mathrm{~mm}$. Two layers of the SMC prepreg were normally used to produce the composite plates for mechanical tests. A temperature of $140{ }^{\circ} \mathrm{C}$ and a pressure of $10 \mathrm{MPa}$ were applied to the material for about 5 minutes. The impregnated material was moulded to a notional thickness of $3.5 \mathrm{~mm}$. Composite samples with different fibre volume fractions were also produced in an exact same way by varying the doctor blade gap in the SMC machine, resulting in different quantities of resin paste being deposited on the fibre mats.

\subsection{Tensile testing}

Tensile tests of hemp fibre SMC composite samples were carried out according to ASTM D 3039-95. The composite specimens were prepared with end tabs to prevent failure close to the grips. The dimensions of the tensile specimens were $220 \mathrm{~mm} \times 25.4 \mathrm{~mm}$ with an average thickness of $3.8 \mathrm{~mm}$. The gauge length was $140 \mathrm{~mm}$. The tensile tests were preformed on an Instron 6025 universal tensile testing machine at a cross-head speed of $1 \mathrm{~mm} / \mathrm{min}$.

\section{Micromechanical modelling}


Cox-Krenchel [35-36] and Kelly-Tyson models [37] are often used to predict the modulus and tensile strength of random fibre reinforced composites. For glass fibre [38-39] and even some natural fibre composites [40] these models give good predictions. However, for most natural fibre reinforced composites these models tend to overestimate the properties, particularly at high fibre content because they do not take into account on the effect of voids in the matrix, the lumen in the plant fibres, the distribution of fibres and additional fillers in the composites and fibre straightness, etc. .

Madsen and Lilholt's [41] work on unidirectional flax/polypropylene composites suggested that the porosity of the composites can be calculated using:

$$
\begin{aligned}
& V_{f}=\frac{1}{1+\alpha} \cdot\left(1-\left(V_{p(\text { proc })}+V_{p(s t r u c)}\right)\right) \\
& V_{m}=\frac{\alpha}{1+\alpha} \cdot\left(1-\left(V_{p(\text { proc })}+V_{p(\text { struc })}\right)\right)
\end{aligned}
$$

where $V_{f}$ and $V_{m}$ are the volume fractions of fibre and matrix, $V_{p(\text { proc })}$ is the porosity component related to the porosities created during composite processing and $V_{p(s t r u c)}$ is the porosity component assigned to structural mechanisms resulting from the limit of compaction of the plant fibres. $\alpha$ is a factor related to the fibre weight fraction:

$$
\alpha=\frac{\left(1-W_{f}\right) \cdot \rho_{f}}{W_{f} \cdot \rho_{m}}
$$

where $W_{f}$ is the fibre weight fraction, $\rho_{f}$ and $\rho_{m}$ is the density of fibre and matrix.

The Cox-Krenchel model for composite stiffness is given by:

$$
E_{c}=\eta_{0} \eta_{L E} V_{f} E_{f}+\left(1-V_{f}\right) E_{m}
$$

where $E_{f}$ and $E_{m}$ is the tensile modulus of fibre and matrix, $V_{f}$ is the fibre volume fraction, $\eta_{0}$ is the fibre orientation factor of $3 / 8$ for two-dimensional random orientation and $1 / 5$ for three-dimensional random orientation. $\eta_{L E}$ is the fibre length efficiency factor and is calculated by:

$$
\eta_{L E}=\left[1-\frac{\tanh (\beta L / 2)}{\beta L / 2}\right]
$$

where 


$$
\beta=\frac{2}{D}\left[\frac{2 G_{m}}{E_{f} \ln \left(\sqrt{\pi} / x_{i} V_{f}\right)}\right]^{1 / 2}
$$

where $L$ is the fibre length, $G_{m}$ is the shear modulus of the matrix and $x_{i}$ is the geometrical packing arrangement of the fibres.

The Kelly-Tyson model for composite strength is given by:

$$
\sigma_{u c}=\eta_{0}\left(\sum_{i}\left[\frac{\tau L_{i} V_{i}}{D}\right]+\sum_{j}\left[\sigma_{j} V_{j}\left(1-\frac{L_{c}}{2 L_{j}}\right)\right]\right)+\left(1-V_{f}\right) \sigma_{m}
$$

where $\eta_{0}$ is the fibre orientation factor, $\tau$ is the interfacial shear strength, $\sigma_{m}$ is the strength of matrix, $L$ and $L_{c}$ is the fibre length and critical fibre length. The first summation is the strength contribution for all fibres of sub-critical length $\left(L\left\langle L_{c}\right)\right.$ and the second summation for fibres of super-critical length $\left(L>L_{c}\right)$. To improve the prediction of strength Shao-Yun et al. [42] introduced an additional fitting parameter $k$ :

$$
\sigma_{u c}=k \eta_{0}\left(\sum_{i}\left[\frac{\tau L_{i} V_{i}}{D}\right]+\sum_{j}\left[\sigma_{j} V_{j}\left(1-\frac{L_{c}}{2 L_{j}}\right)\right]\right)+\left(1-V_{f}\right) \sigma_{m}
$$

The theoretical effect of porosity on the material stiffness [43] can be given by:

$$
E_{p}=E_{d} \cdot\left(1-V_{p}\right)^{2}
$$

Where the subscripts $d$ and $p$ represent the fully dense material and the porous material, respectively. Now applying Equation (9) to Cox-Krenchel and Kelly-Tyson models gives:

\section{Modified Cox-Krenchel}

$$
E_{c}=\eta_{0} \eta_{L E} V_{f} E_{f}+\left(1-V_{f}\right) E_{m} \cdot\left[1-V_{p}\right]^{2}
$$

Modified Kelly-Tyson

$$
\sigma_{u c}=k \eta_{0}\left(\sum_{i}\left[\frac{\tau L_{i} V_{i}}{D}\right]+\sum_{j}\left[\sigma_{j} V_{j}\left(1-\frac{L_{c}}{2 L_{j}}\right)\right]\right)+\left(1-V_{f}\right) \sigma_{m} \cdot\left[1-V_{p}\right]^{2}
$$




\section{Results and Discussion}

\subsection{Fibre morphology}

Scanning electron microscopy (SEM) was used to examine the change in the surface morphology of the surface treated hemp fibres. A SEM micrograph of an untreated hemp fibre is shown in Figure 2(a). The micrographs show that the untreated fibres have a smooth surface, possibly due to the wax and lignin substances on the fibre surface [44]. The SEM image shows that hemp fibres have a circular diameter and that the thickness of the fibres is not uniform but varies along the fibre length. The SEM micrograph of an alkaline treated fibre is shown in Figure 2(b). The surface of the fibre has become rough and micro-ridges are visible along the fibre length. The increase in the roughness of the fibre surface after alkaline treatment may be partly due to the removal of the wax layer [45]. This increased roughness enhances the possibility for mechanical interlocking at the interface. The micro-ridges resulting from the separation of the fibres into single fibrils could be due to the removal of lignin material that acts as glue holding individual fibrils together. A SEM micrograph of a silane treated fibre is shown in Figure 2(c). Also here grooves and micro-ridges are observed and the silane treatment is for building-up the chemical links between hydrophebic surface of the fibre and hydrophobic polyester matrix.

\subsection{Influence of fibre content on mechanical properties \\ 4.2.1 Tensile strength}

Figure 3 shows the tensile strength results of H-SMC and G-SMC composites as a function of fibre volume fraction, together with the Kelly-Tyson (Eq. 8) and porosity modified Kelly-Tyson (Eq. 11) predictions for H-SMC composites. A constant filler content of 18 vol.\% was used. For the Kelly-Tyson model the following parameters are used: fibre strength $\left(\sigma_{\mathrm{f}}\right)$ of $650 \mathrm{MPa}$ [46], fibre length $\left(\mathrm{L}_{\mathrm{f}}\right)$ of $65 \mathrm{~mm}$, interfacial shear strength $(\tau)$ of $14 \mathrm{MPa}$ [47], matrix strength at fibre failure strain of $5.13 \mathrm{MPa}\left(\mathrm{E}_{\mathrm{m}} \sigma_{\mathrm{f}} / \mathrm{E}_{\mathrm{f}}=\right.$ $(2000 / 690) / 26900)$, critical fibre length $\left(\mathrm{L}_{\mathrm{c}}\right)$ of $26.5 \mathrm{~mm}\left(\sigma_{\mathrm{f}} \mathrm{D} / 2 \tau=690 * 0.022 /(2 * 14)\right)$, fibre orientation factor $\left(\eta_{0}\right)$ of $3 / 8$ and a fitting efficiency parameter $(k)$ of 0.18 . A similar efficiency parameter of 0.2 was used by Garkhail et al. [40] for flax/PP composites. It is clear from the graph that the reinforcing efficiency of hemp fibres is less than those of glass fibres. The reason for this could be the non-uniformity of natural fibres resulting in large scatter in fibre strength. Another possible reason could be fibre anisotropy resulting in transverse hemp fibre bundle failure. The hemp fibre mat was made of chopped fibre bundles (technical fibres) which consist of many smaller elementary fibres glued together by a weak 
pectin interface. The surface treatment only modifies the fibre bundle surface but not the interfaces between the elementary fibres within the fibre bundle. The failure of the composite is therefore expected to be initiated not only at the fibre-matrix interface but also at the elementary fibre-pectin interfaces within the fibre bundle. Cracks will propagate not only through the fibre-matrix interface, but also through the interfaces between the elementary fibres [48]. Therefore further optimisation of the fibrematrix interface through surface treatments will have limited influence on the composite mechanical properties. This is displayed schematically in Figure 4. A higher efficiency parameter (k) of 0.46 for GSMC was obtained, possibly because in these isotropic fibres such a failure mode within the fibre cannot occur. G-SMC composite show a good agreement with Kelly-Tyson predictions for all fibre volume fractions, while H-SMC composites have a relatively good agreement at low fibre volume fractions while at high fibre volume fractions the experimental values are considerably lower then the prediction. This is due to the relatively high porosity content in natural fibre composites at high fibre volume fraction which the Kelly-Tyson model does not take into account. H-SMC composites have good agreement with the modified Kelly-Tyson prediction for tensile strength at all fibre volume fractions; since this model includes the contribution of porosity content on tensile strength.

The specific properties and price/performance index of G-SMC and H-SMC composites at three different fibre volume fractions are given in Table 1. At lower fibre content the specific strength of GSMC is slightly higher then H-SMC while at higher fibre content the specific strength of H-SMC is higher then G-SMC. A similar trend is noticed for the price/performance index with H-SMC composites having a better price/performance ratio then G-SMC composites at higher fibre loadings. The results indicate that $\mathrm{H}-\mathrm{SMC}$ composites should be manufactured with relatively high hemp fibre contents to achieve maximum benefits in terms of higher specific properties and price/performance index compared to G-SMC composites.

\subsubsection{Tensile modulus}

Figure 5 shows the tensile modulus of H-SMC and G-SMC composites as a function of fibre volume fraction, together with the Cox-Krenchel (Eq. 4) and modified Cox-Krenchel (Eq. 10) predictions for H-SMC composites. A constant filler content of 18 vol.\% was used. For the Cox-Krenchel model the following parameters are used: fibre modulus $\left(\mathrm{E}_{\mathrm{f}}\right)$ of $26.9 \mathrm{GPa}$ [46], matrix modulus $\left(\mathrm{E}_{\mathrm{m}}\right)$ of $3.8 \mathrm{GPa}$, geometrical packing arrangement of fibre of 4 , shear modulus of the matrix $\left(\sigma_{\mathrm{m}}\right)$ is $0.71 \mathrm{GPa}\left(\mathrm{E}_{\mathrm{m}} / 2(1+v)=\right.$ $2 / 2(1+0.4))$, a fibre length $\left(L_{f}\right)$ of $65 \mathrm{~mm}$ and fibre orientation factor $\left(\eta_{\mathrm{o}}\right)$ of $3 / 8$. Based on the results it 
can be concluded that the stiffness of H-SMC composites is comparable to G-SMC composites at low fibre volume fractions, while at higher fibre loadings the modulus of H-SMC composites is slightly lower then that of G-SMC. However, the specific stiffness of H-SMC composites is similar to G-SMC composites (see Table 1) considering the low density of hemp fibres $\left(1.5 \mathrm{~g} / \mathrm{cm}^{3}\right.$ for hemp compared to 2.5 $\mathrm{g} / \mathrm{cm}^{3}$ for E-glass).

However, the price/performance index for tensile modulus is fairly similar for both H-SMC and G-SMC. Again the G-SMC composites have a good agreement with the Cox-Tyson prediction at all fibre volume fractions, while the H-SMC composites only show a good agreement with Kelly-Tyson predictions at low fibre volume fractions. H-SMC composites show good agreement with the modified Cox-Tyson prediction at all volume fractions.

The models for strength and modulus predictions can be further improved if the porosity content of the composite and other factors of the hemp fibre such as strength and length can be measured more accurately. The results indicate that further research should be focused on porosity reduction for the HSMC composites by improved processing and compaction methods.

\subsection{Influence of $\mathrm{CaCO}_{3}$ content on mechanical properties}

Figure 6 shows the dependence of the tensile strength and modulus of H-SMC composites on the $\mathrm{CaCO}_{3}$ filler content at a constant fibre volume fraction of $23 \%$. Interestingly, the strength of the composite increases with filler content. An improvement of $14 \%$ in tensile strength of the composite is obtained with the addition of 26 vol. $\%$ filler content compared to the unfilled composite specimens. At filler content of 35 vol.\% there seems to be a slight decrease in composite strength, while the stiffness of the composites increases continuously with increasing filler content. Addition of 35 vol.\% filler content resulted in a $16 \%$ increase in the modulus of the composite. The specific properties and price/performance index of $\mathrm{H}-\mathrm{SMC}$ composites at increasing $\mathrm{CaCO}_{3}$ filler content is given in Table 2 . The specific properties of $\mathrm{H}-\mathrm{SMC}$ composites decrease with increasing $\mathrm{CaCO}_{3}$ filler content. This was expected as calcium carbonate has a relatively high density of $2.71 \mathrm{~g} / \mathrm{cm}^{3}$. An alternative to using $\mathrm{CaCO}_{3}$ as a filler in H-SMC composites could be to use rice husk powder (RHP). Thanomsilp et al. [49] on their work on RHP as a substitute for $\mathrm{CaCO}_{3}$ fillers for glass fibre/polyester moulding compounds showed that weight-for-weight, the composites containing RHP exhibit better or at least comparable mechanical properties to those containing $\mathrm{CaCO}_{3}$ filler. As expected, generally the price/performance index for 
tensile strength and modulus of $\mathrm{H}-\mathrm{SMC}$ composites increases with increasing $\mathrm{CaCO}_{3}$ filler content because of the relatively low cost of $\mathrm{CaCO}_{3}$ filler.

The fractured surfaces of tensile tested $\mathrm{H}-\mathrm{SMC}$ composites with different $\mathrm{CaCO}_{3}$ filler content are shown in Figure 7. The fracture surface of the unfilled composite (Figure 7a) shows a smooth and overlapping matrix phase with sharp edges, while the fracture surface of 8 vol. $\% \mathrm{CaCO}_{3}$ filled composite (Figure $7 b$ ) shows a high level of porosity and the fibres are well imbedded in the matrix. The fractured surface of the composite with 26 vol.\% filler (Figure 7c) shows that fibres are again well imbedded in the matrix but the porosity content is considerably low. This decrease in porosity content is presumably responsible for the increase in tensile strength. The fractured surface of composites with 35 vol.\% $\mathrm{CaCO}_{3}$ filler content (Figure 7d) shows that the wetting of the fibre becomes poor which results in the reduction in tensile strength. Finally, it is concluded that the filler content of $25-30$ vol. $\%$ is the optimal to obtain a good balance in tensile strength and stiffness for H-SMC composites.

\subsection{Influence of hemp fibre surface treatment}

The tensile strength and stiffness of the H-SMC composites based on hemp fibre with different fibre surface treatments are presented in Figures 8 and 9. In this study, three kinds of hemp fibre surface treatments were used: (i) for fibre cleaning (de-waxing and bleaching) an alkaline $(\mathrm{NaOH})$ treatment was used, (ii) for fibre coating (grafting and coupling) a silane treatment was used and (iii) additionally a combination of these two treatments (constant $2 \% \mathrm{NaOH}$ concentration with varying silane concentration) was also investigated.

\subsubsection{Alkaline Treatment}

The tensile strength of the composites increases with alkaline treatment up to a concentration of $2 \%$. However, further increase in alkaline concentration resulted in a decrease in tensile strength. There was, however, a significant improvement of $60 \%$ to the tensile strength for composites incorporating hemp fibres treated with $2 \%$ alkaline solution compared to untreated composites. A similar trend to the tensile modulus is observed for the composite, with an increase in stiffness by $2 \% \mathrm{NaOH}$ solution treatment , followed by a reduction at higher concentration treatment. An improvement of 53\% in composite stiffness is obtained for specimens treated with $2 \% \mathrm{NaOH}$ compared to untreated specimens. As can be seen in Figure 2(b), the treatment of the hemp fibres with $\mathrm{NaOH}$ solution removed the impurities and waxy like substances on the fibre surface, thereby making the topography of the fibre 
surface rougher. This probably enhanced the mechanical interlocking between the fibre and matrix resulting in an increased tensile strength for alkaline treated fibre composites. Ganan et al. [50] suggested that removal of amorphous components, such as lignin and surface impurities could enhance wetting due to more hydrogen bonds being formed between the purified cellulose fibre surface and the matrix.

\subsubsection{Silane Treatment}

The tensile strength of the H-SMC composites increases with increasing silane concentration up to a concentration of $2 \%$, beyond which the strength does not increase any further. An improvement of $32 \%$ in tensile strength is achieved for composites treated with $2 \%$ silane with respect to the untreated composites. Unlike tensile strength, the composite stiffness continues to increase with increasing silane concentration. An improvement of $24 \%$ in stiffness is obtained for composites treated with $5 \%$ silane solution.

\subsubsection{Alkaline-Silane Treatment}

The most significant improvement in tensile strength and stiffness of $80 \%$ and $82 \%$, respectively is achieved by a combination of alkaline and silane treatments. The reason for the large increase in tensile properties is that the alkaline treatment as mentioned above removed the impurities on the hemp fibre surface resulting in more active sites or fresh surfaces for bonds to form with the silane molecules. In this case much more bonds can be formed chemically between the cellulose hydroxyl groups on the fibre surface and the silane molecules than that of without the alkaline treatment.

\subsection{Fracture surface analysis}

The tensile fractured surfaces of untreated and treated H-SMC composites were studied with scanning electron microscopy (SEM). Figure 10(a) shows the fractured surface of an untreated H-SMC composite. It shows that the surface of the fibres is clean, with little traces of resin adhering to them, suggesting poor adhesion of the polyester resin to the hemp fibres. Furthermore, considerable fibre pullout is observed in the micrographs of the untreated composite. A SEM fracture surface of an alkaline treated hemp fibre composite is shown in Figure 10(b). Traces of resin are noticed on the fibre surface, while the imbedded fibres are completely surrounded by matrix and fewer gaps are visible between fibre and matrix. This suggests better wetting of the hemp fibres by the polyester resin and considerably less fibre pull-out and debonding is observed. The fracture surfaces of alkaline treated H-SMC composites show that fibrillation of the fibre bundles has occurred, particularly for $2 \% \mathrm{NaOH}$ concentrations and 
above. A SEM micrograph of the silane treated fibre composite is shown in Figure 10(c). The exposed fibre is clearly covered by residue of resin, therefore implying good wetting of the fibre by the polyester resin. Again considerably less fibre pull-out is noticed for the silane treated fibre composite compared to the untreated fibre composite. Again this indicates that silane treatment has enhanced the fibre/matrix adhesion. Furthermore, like the alkaline treated fibre composites the main failure mechanism of the silane treated fibre composites is fibrillation. The fractured surfaces of alkaline-silane treated composites show similarly features to the alkaline and silane fractured surfaces and therefore are not presented here.

\section{Conclusions}

SMC composites were made using random hemp fibre mat and unsaturated polyester SMC resin system. Material parameters that were studied for the optimization of the mechanical performance of the H-SMC composites covered fibre volume fraction, filler volume fraction and interfacial modification through the use of alkaline and silane surface treatments. In order to understand the performance of these H-SMC composites better, they were compared with the properties of glass fibre reinforced composites (G-SMC) which were manufactured in a similar manner. The mechanical performance of the H-SMC was evaluated using modified versions of "Cox-Krenchel" and "Kelly-Tyson" models which included the effect of composite porosity. A rather good agreement between these modified models and the experimental data was found for both composite tensile strength and stiffness. A significant effect of fibre volume fraction and $\mathrm{CaCO}_{3}$ filler content on the natural fibre composite mechanical properties was found and established. The combination of alkaline and silane treatment on the hemp fibre resulted in a significant increase in mechanical properties.

The results demonstrate that the mechanical properties of $\mathrm{H}-\mathrm{SMC}$ are comparable to G-SMC composites especially when considering the low density of hemp fibres compared to glass fibres. Additionally, potential cost savings associated with the H-SMC composites are very significant both in terms of raw materials and disposal after life-time, which make them a rather attractive alternative to glass fibre based SMCs.

To make further improvements in the mechanical performance of the H-SMC composites future research should be mainly focused on reducing the porosity content in the H-SMC composites.

\section{Acknowledgements}


The authors are grateful to Building Research Establishment (BRE) and Engineering and Physical Sciences Research Council (EPSRC) for funding the present study. Thanks also to Dr Mizi Fan (BRE) for his help and assistance. We are grateful to Menzolit Ltd, UK for allowing us to use the SMC equipment and providing the raw materials. The help and assistance in the preparation of the composites, as well as stimulating discussions with Mr. Steve Crowther are also very gratefully acknowledged.

\section{References}

1. M. Revellino, L. Saggese and E. Gaiero: Compression moulding of SMCs. Comprehensive Composite Materials, 2000, 2, 763.

2. S. Goutianos, T. Peijs, B. Nystrom and M. Skrifvars: Appl. Compos. Mater., 2006, 13(4), 199.

3. A. Stamboulis, C.A. Baillie, S.K. Garkhail, H.G.H. Van Melick and T. Peijs: Appl. Compos. Mater., $2000,7,273$.

4. J. George, E.T.J. Klompen and T. Peijs: Adv. Compos. Lett., 2001, 10(2), 81.

5. A.C.N. Singleton, C.A. Baillie, P.W.R. Beaumont and T. Peijs: Compos. Part B, 2003, 42(6), 519.

6. T. Peijs: Mater. Technol., 2000, 15(4), 281.

7. D. Rouison, M. Couturier, M. Sain, B. MacMillan and B.J. Balcom: Polym. Compos., 2005, 26(4), 509.

8. A. Stamboulis, C.A. Baillie and T. Peijs: Compos. Part A, 2001, 32(8), 1105.

9. T. Peijs, S. Garkhail, R. Heijenrath, M. van den Oever and H. Bos: Macromolecular Symposia, 1998, 57, 193.

10. K. Oksman: Appl. Compos. Mater., 2000, 7, 403.

11. T.D. Hapuarachchi, G. Ren, P.J. Hogg and T. Peijs: Appl. Compos. Mater., 2007, 14(4), 251.

12. P. Wambua, J. Ivens and I. Verpoest: Compos. Sci. Technol., 2003, 63(9), 1259.

13. A. Arbelaiz, B. Fernandez, G. Cantero, R. Llano-Ponte, A. Valea and I. Mondragon: Compos. Part A, 2005, 36(12), 1637.

14. P.W.J. Van den Heuvel, Y.J.W. Van der Bruggen and T. Peijs: Compos. Part A, 1996, 27(9), 855.

15. R.W. Venderbosch, T. Peijs, H.E.H. Meijer and P.L. Lemstra: Compos. Part A, 1996, 27(9), 895.

16. M. Van den Oever and T. Peijs: Compos. Part A, 1998, 29(3), 227.

17. M.S. Madhukar and L.T. Drzal: J. Compos. Mater., 1991, 25(8), 958.

18. K. Joseph, L.H.C. Mattoso, R.D. Toledo, S. Thomas, L.H. de Carvalho, L. Pothen, S. Kala and B. James: Natural Polymers and Agrofibers Composites, Embrapa, San Carlos, Agrofibres Compos. $2000,159$.

19. S. Mishra, M. Misra, S.S. Tripathy, S.K. Nayak and A.K. Mohanty: Polym. Compos., 2002, 23(2), 164.

20. M.S. Sreekala, M.G. Kumaran, S. Joseph, M. Jacob and S. Thomas: Appl. Compo. Mater., 2000, 7, 295.

21. C. Qui, N. Soykeabkaew, Xiuyuan and T. Peijs: Carbohydrate Polym., 2008, 71(3), 458.

22. T.T.L. Doan, S.L. Gao, E. Mader: Compos. Sci. Technol., 2006, 66, 952. 
23. A. Murkhejee, P.K. Ganguly and D. Sur: J Textile Institute, 1993, 84(3), 348.

24. P.J. Herrera-Franco and G.A. Valadez: Compos. Part A, 2004, 35, 339.

25. I. Van de Weyenberg, J. Ivens, A. De Coster, B. Kino, E. Baetens and I. Verpoest: Compos. Sci. Technol., 2003, 63, 1241.

26. S. Aziz, M.P. Ansell, J.S. Clarke and S.R. Panteny: Compos. Sci. Technol., 2005, 65(3-4), 525.

27. L.U. Devi, S.S. Bhagawan and S. Thomas: J Appl. Polym. Sci,. 1997, 64(9), 1739.

28. J. Gassan, I. Mildner and A.K. Bledzki: Mech. Compos. Mater., 1999, 35, 653.

29. D.G. Hepworth, D.M. Bruce, J.F.V. Vincent and G. Jeronimidis: J Mater. Sci., 2000, 35, 293.

30. S. Goutianos and T. Peijs: Adv. Compos. Lett., 2003, 12(6), 237.

31. A.C. Wibowo, A.K. Mohanty, M. Misra and L.T. Drzal: Indus. Eng. Chem. Res., 2004, 43(16), 4883.

32. S. Luo: J. Mater. Sci., 1999, 34(15), 3709.

33. N. Soykeabkaew, N. Arimoto, T. Nishino and T. Peijs: Compos. Sci. Technol., 2008, 68(10-11), 2201.

34. R. Heijenrath and T. Peijs: Adv. Compos. Lett., 1996, 5(3), 81.

35. H.L. Cox: British J. Appl. Phy., 1952, 3, 72.

36. H. Krenchel: In Fibre Reinforced, Akademisk Forlad, 1964, Copenhagen.

37. A. Kelly and W.R. Tyson: J. Mech. \& Phy. Solids, 1965, 13, 329.

38. J.L. Thomason and W.M. Groenewoud: Compos. Part A, 1996, 27A, 555.

39. J.L. Thomason, M.A. Vlug, G. Schipper and H.G.L.T. Krikor: Compos. Part A, 1996, 27A, 1075.

40. S.K. Garkhail, R.W.H. Heijenrath and T. Peijs: Appl. Compos. Mater., 2000, 7, 351.

41. B. Madsen and H. Lilholt: Compos. Sci. Technol., 2003, 63, 1265.

42. F. Shao-Yun and L. Bernd: Compos. Sci. Technol., 1996, 56, 1179.

43. J.K. MacKenzie: Proceedings Physical Society, 1950, B63, 2.

44. N.E. Zafeiropoulos, D.R. Williams, C.A. Baillie and F.L. Mattews: Compos. Part A, 2002, 33, 1083.

45. M.S. Sreekala, M.G. Kumaran and S. Thomas: J. Appl. Polym. Sci., 1997, 66, 821.

46. A.K. Bledzki and J. Gassan: Progress in Polym. Sci., 1999, 24, 221.

47. C. Baley, F. Busnel, Y. Grohens and O. Sire: Compos. Part A, 2006, 37, 1626.

48. H. Bos: The potential of flax fibres as reinforcement for composite materials. PhD thesis 2004 Eindhoven University of Technology, Eindhoven.

49. C. Thanoslip, S. Cauchi-Savona, T. Peijs and S. Posyachinda: J. Biobased Mater. \& Bioenergy, 2007, $\mathbf{1}(1), 87$.

50. P. Ganan, S. Garbizu, R. Llano-Ponte and I. Mondragon: Polym. Compos., 2005, 26, 121.

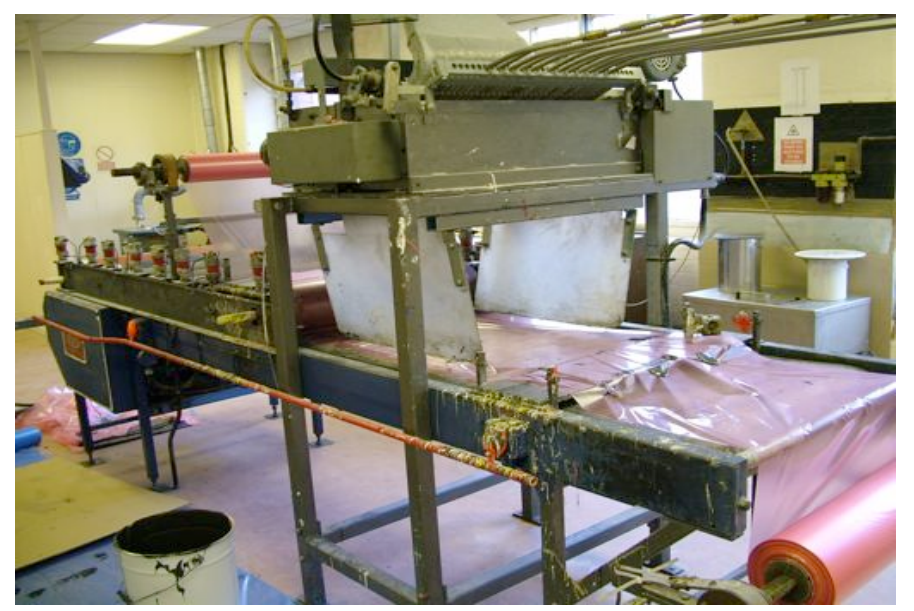


Figure 1

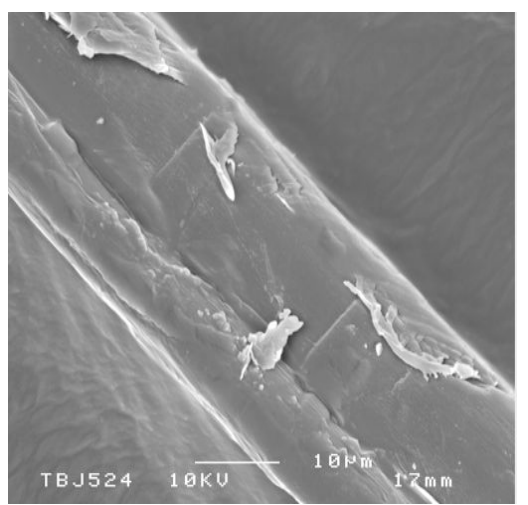

(a)

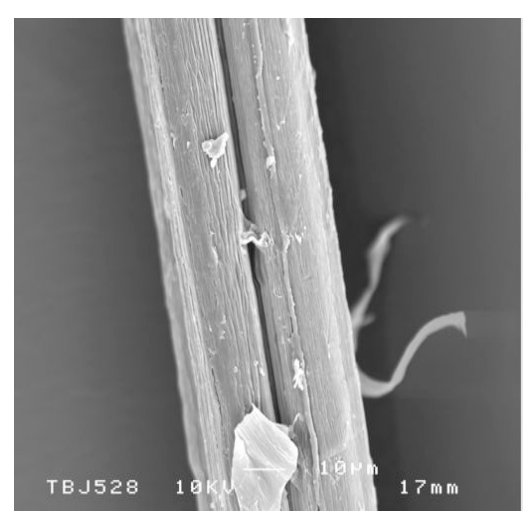

(b)

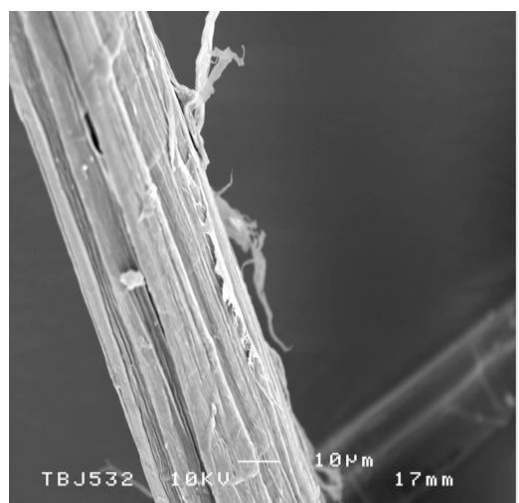

(c)

Figure 2

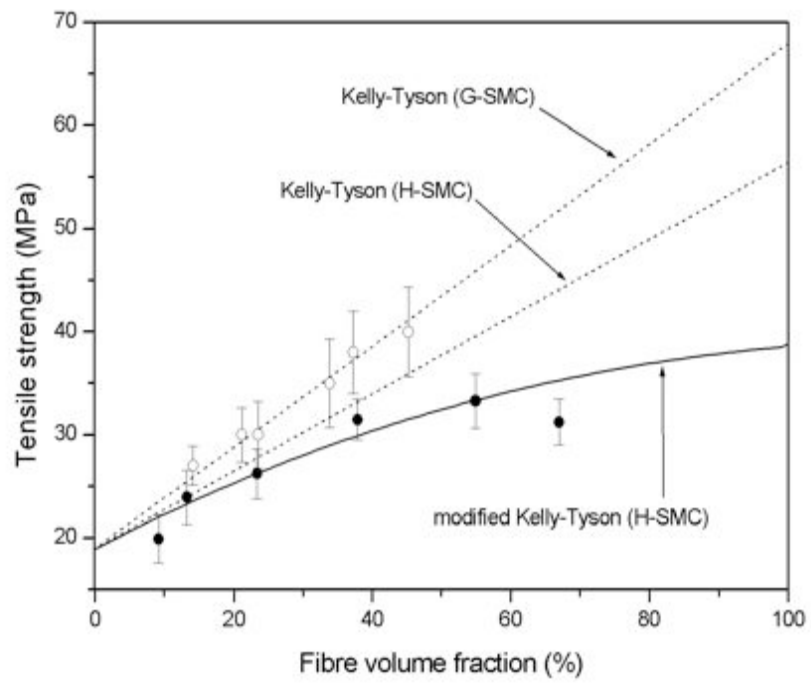

Figure 3

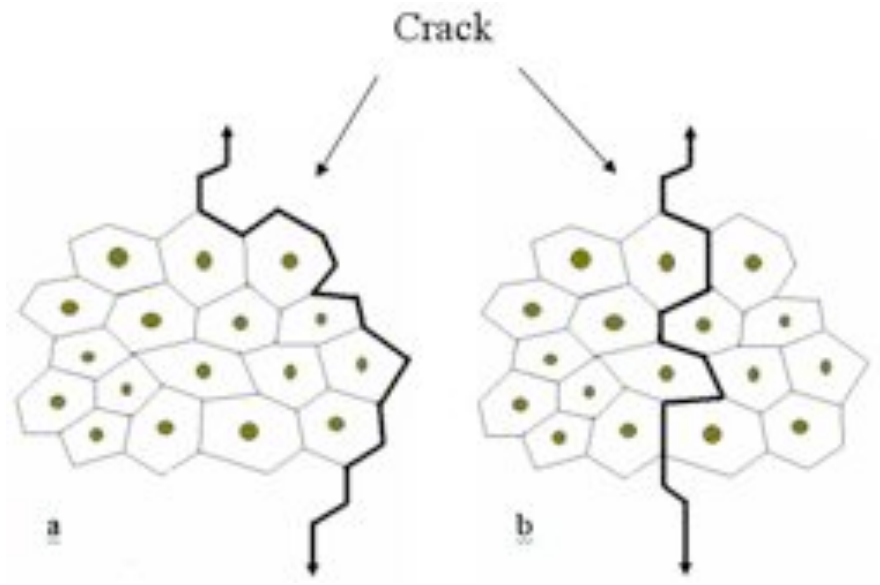

Figure 4 


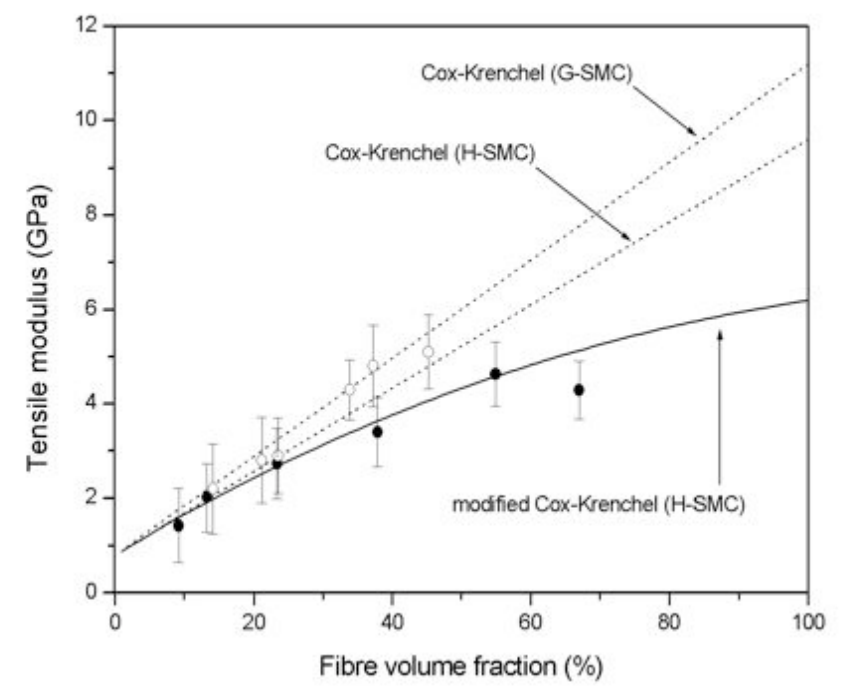

Figure 5

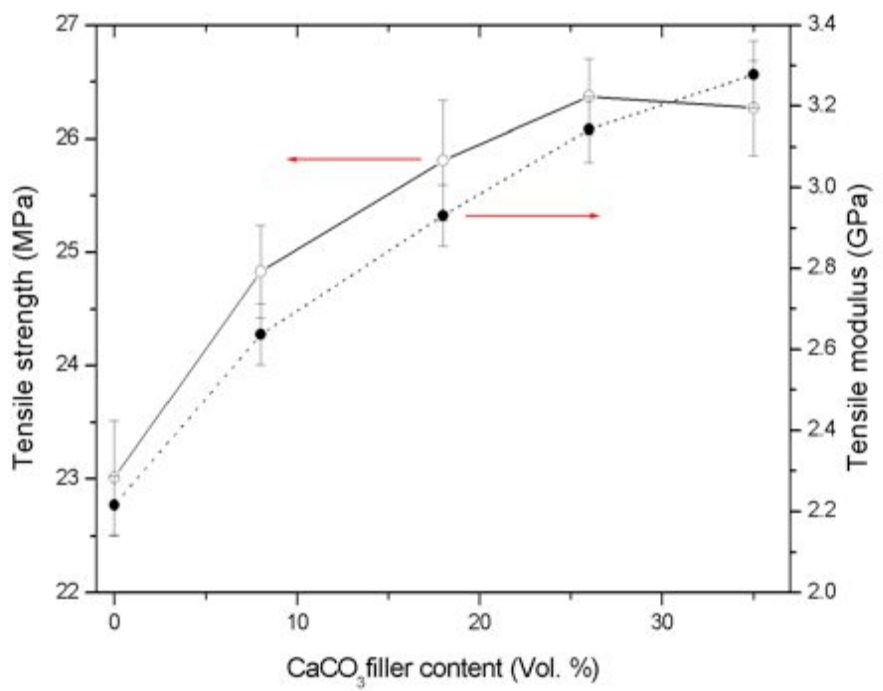

Figure 6
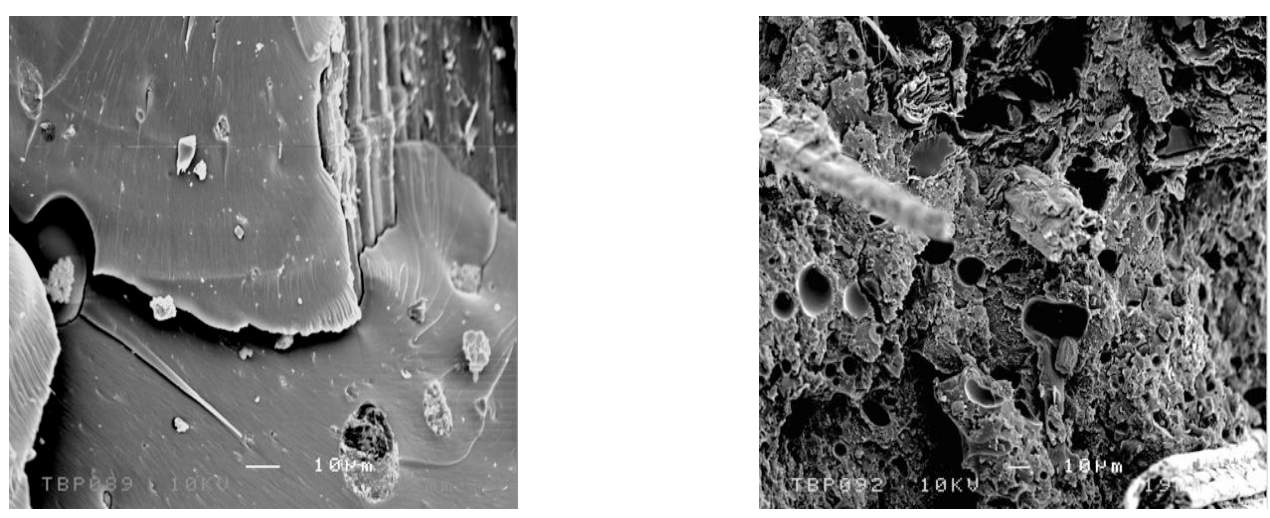
(a)

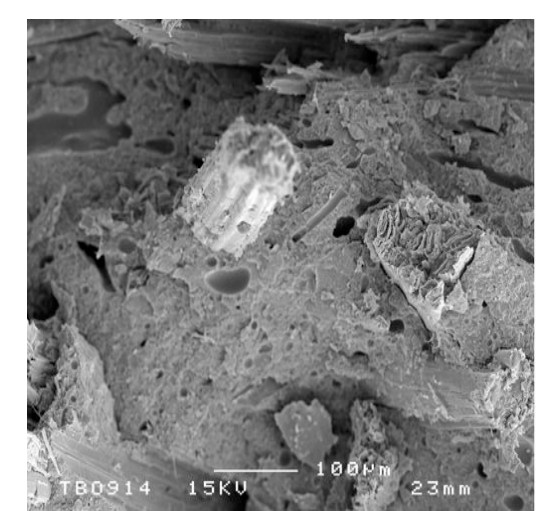

(c) (b)

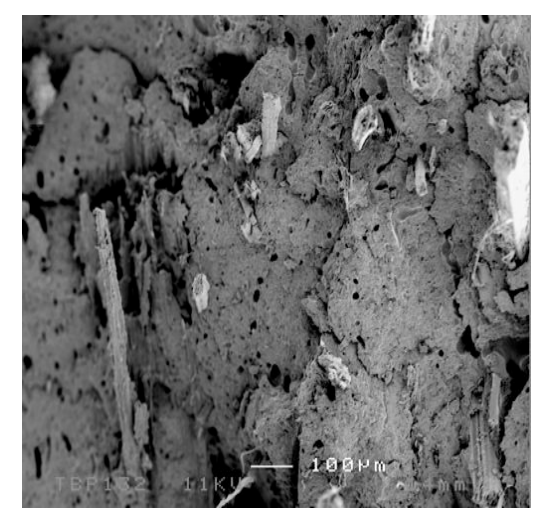

(d)

Figure 7

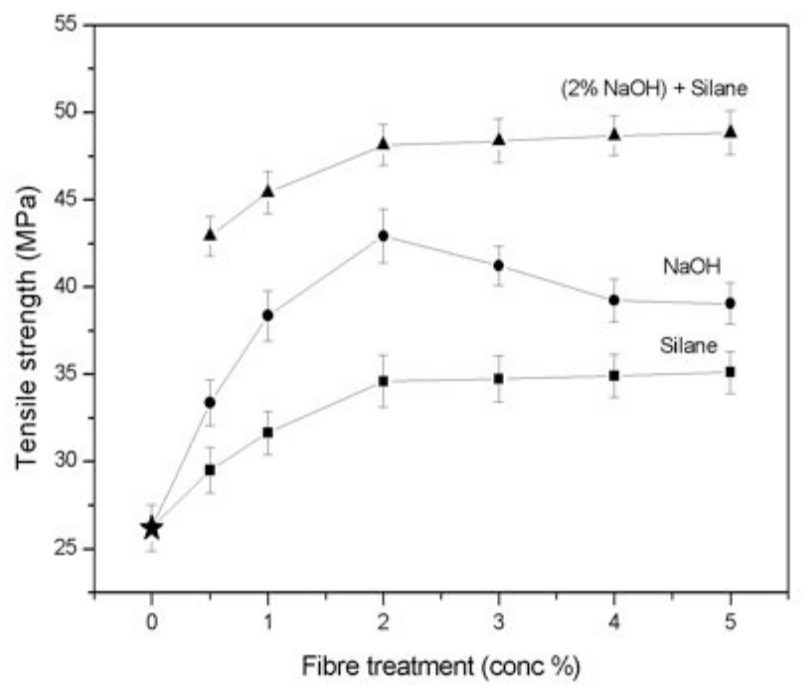

Figure 8

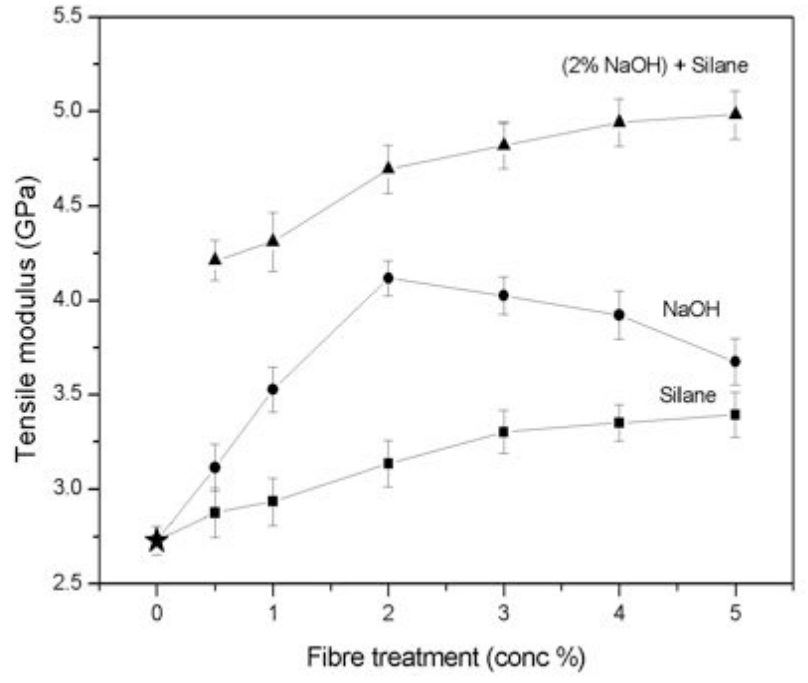

Figure 9 


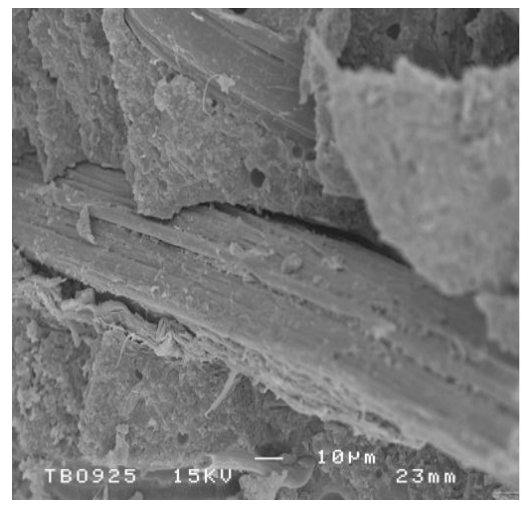

(a)

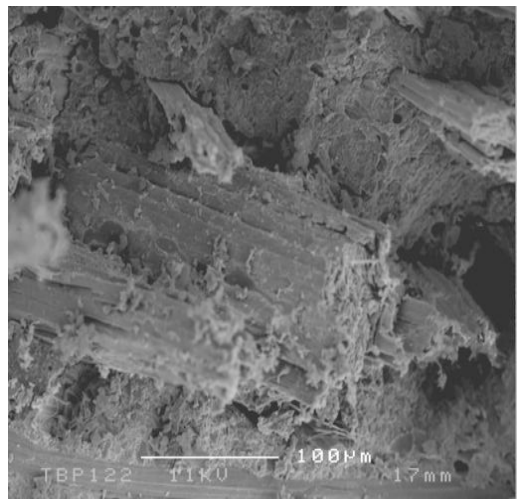

(b)

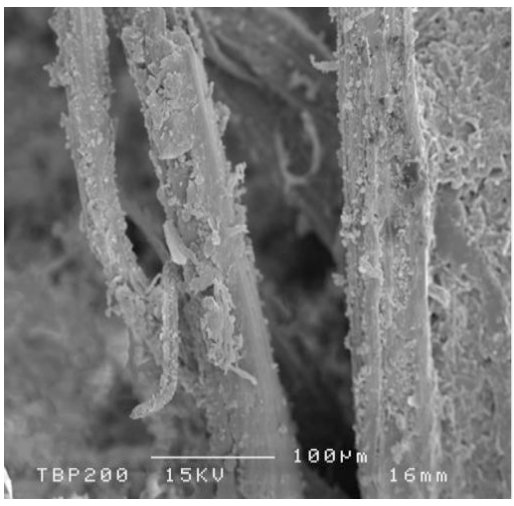

(c)

Figure 10

Table 1

\begin{tabular}{|c|c|c|c|}
\hline & Fibre vol.\% & $G-S M C$ & $H-S M C$ \\
\hline \multirow{3}{*}{ Specific strength $\left(\mathrm{MPa} / \mathrm{gcm}^{-3}\right)$} & 13 & 13.17 & 12.47 \\
\hline & 23 & 14.22 & 14.24 \\
\hline & 37 & 19.00 & 19.78 \\
\hline \multirow{3}{*}{ Specific modulus $\left(\mathrm{GPa} / \mathrm{gcm}^{-3}\right)$} & 13 & 1.07 & 1.05 \\
\hline & 23 & 1.37 & 1.48 \\
\hline & 37 & 2.40 & 2.14 \\
\hline \multirow{3}{*}{ Strength/Price $\left(\sigma / € \mathrm{~m}^{-2}\right)^{*}$} & 13 & 4.86 & 5.96 \\
\hline & 23 & 6.09 & 6.22 \\
\hline & 37 & 5.54 & 6.07 \\
\hline \multirow{3}{*}{ Modulus/Price $\left(E / € m^{-2}\right)^{*}$} & 13 & 0.50 & 0.50 \\
\hline & 23 & 0.59 & 0.65 \\
\hline & 37 & 0.76 & 0.66 \\
\hline
\end{tabular}

* Hemp and glass fibre mat prices according to J. Hobso, Hemcore Ltd and S. Crowther, Menzolit Ltd.

Table 2

\begin{tabular}{c|cccc}
\hline $\begin{array}{c}\text { Filler volume } \\
\text { fraction (vol.\%) }\end{array}$ & $\begin{array}{c}\text { Specific strength } \\
\left(\mathbf{M P a} / \mathbf{g c m}^{-3}\right)\end{array}$ & $\begin{array}{c}\text { Specific modulus } \\
\left(\mathbf{G P a} / \mathbf{g c m}^{-3}\right)\end{array}$ & $\begin{array}{c}\text { Strength/Price } \\
\left(\mathbf{\sigma} / \mathbf{E m}^{-2}\right)\end{array}$ & $\begin{array}{c}\text { Modulus/Price } \\
\left(\mathbf{E}_{\mathbf{E m}} \mathbf{m}^{-2}\right)\end{array}$ \\
\hline $\mathbf{0}$ & 20.54 & 1.98 & 6.07 & 0.58 \\
\hline $\mathbf{8}$ & 18.53 & 1.97 & 6.45 & 0.68 \\
\hline $\mathbf{1 8}$ & 16.03 & 1.82 & 6.57 & 0.75 \\
\hline $\mathbf{2 6}$ & 14.49 & 1.73 & 6.61 & 0.79 \\
\hline $\mathbf{3 5}$ & 12.69 & 1.58 & 6.47 & 0.81 \\
\hline
\end{tabular}

Figure 1. Photograph of the machine used to fabricate the SMC composites.

Figure 2. SEM micrographs of different hemp fibres: (a) untreated (b) alkaline (NaOH) treated, (c) silane treated.

Figure 3. Tensile strength of $(\bullet)$ H-SMC composites and (o) G-SMC composites as a function of fibre volume fraction. The dotted lines represent the Kelly-Tyson model predictions for G-SMC and H-SMC and the solid line the modified Kelly-Tyson model predictions for H-SMC. 
Figure 4. Schematic of cross section of a fibre bundle (technical fibre) in a matrix containing a crack. (a) The crack propagating through the fibre-matrix interface. (b) The crack propagating through the interfaces between the elementary fibres. Redrawn from Bos [48].

Figure 5. Tensile modulus of $(\bullet) \mathrm{H}-\mathrm{SMC}$ composites and (o) G-SMC composites as a function of fibre volume fraction. The dotted lines represent the Kelly-Tyson model predictions for G-SMC and H-SMC and the solid line the modified Kelly-Tyson model predictions for H-SMC.

Figure 6. (o) Tensile strength and (•) Young's modulus as a function of $\mathrm{CaCO}_{3}$ filler content of $\mathrm{H}-\mathrm{SMC}$ composites.

Figure 7. SEM micrographs of tensile fracture surface of H-SMC composites with (a) 0 vol.\% (b) 8 vol.\% (c) 26 vol. $\%$ and (d) 35 vol. $\% \mathrm{CaCO}_{3}$ filler content/volume fraction at a constant fibre volume fraction of 23 vol. $\%$.

Figure 8 . Tensile strength of $(\star)$ untreated, $(\bullet)$ alkaline treated, $(\mathbf{\square})$ silane treated H-SMC composites as a function of surface treatment. $(\boldsymbol{\Delta})$ represents H-SMC composites based on hemp fibres treated with a fixed $2 \%$ alkaline treatment and varying concentration of silane treatment.

Figure 9. Tensile modulus of $(\star)$ untreated, $(\bullet)$ alkaline treated, ( $\boldsymbol{(})$ silane treated H-SMC composites as a function of surface treatment. $(\boldsymbol{\Delta})$ represents H-SMC composites based on hemp fibres treated with a fixed $2 \%$ alkaline treatment and varying concentration of silane treatment.

Figure 10. SEM micrographs of tensile fracture surface of H-SMC composites (a) untreated (b) alkaline treated and (c) silane treated.

Table 1. Specific properties and price/performance index of G-SMC and H-SMC composites at three different fibre volume fractions.

Table 2. Specific properties and price/performance index of G-SMC and H-SMC composites at different $\mathrm{CaCO}_{3}$ filler content. 